\title{
SIMULATOR-BASED LEARNING: ACHEIVING PERFORMANCE IMPROVEMENT INDEPENDENT OF INSTRUCTORS
}

\author{
Darrell R. Turpin, Reginald T. Welles, and Camille Price \\ Applied Simulation Technologies \\ Salt Lake City, Utah, USA \\ E-mail: dturpin@appliedsimtech.com
}

\begin{abstract}
Summary: The Utah Department of Public Safety (UDPS) implemented a new driver training methodology in 2005, which they have used to train over 950 lawenforcement personnel over the past two years (2005-2006). The new methodology uses driving simulators for whole task practice and for measuring driver-performance. It places simulator-based practice between initial classroom instruction and test-track driver training. Prior analyses of the data collected for 355 drivers in the first year demonstrated that UDPS had successfully achieved: (1) objective measures of driver performance to establish pass/fail criteria, (2) effective motivation for drivers to improve their own performance, and (3) measures of the overall effectiveness of the training process. Summary reports of the first year of data collected at UDPS demonstrated: (1) SAS mitigation levels below the occurrence of test-track discomfort, and (2) a $67 \%$ reduction in risk for collisions by reduction of critical errors. The above success led to expanding the training to $\mathbf{5 9 8}$ new trainees in the second year. This paper reviews new driver performance data collected during 2006. Two additional trainers participated in that process. Analysis of this new data not only confirms the first year's analysis, but newly establishes that: (1) improvement in driver performance is independent of the trainer(s), and (2) training benefit is a function of the sequence and number of exercises.
\end{abstract}

\section{BACKGROUND}

The Utah Department of Public Safety (UDPS) determined that the way they used driving simulators from 2002 to 2004 was not inherently effective in imparting the driving skills that trainees needed to learn in order to cope with the challenges of emergency vehicle operation (EVO). They determined that "game-based situational-awareness exercises" did not impart the required driving skills and could not measure the required skills objectively (Hadley, 2003).

A team at Applied Simulation Technologies (AST) addressed those issues by developing a new course for emergency vehicle operator (EVO) training called EVOC-101. This new course implemented several key principles of instructional systems design (ISD). It provided trainees with practice in applying incrementally advanced safety skills through a sequence of training exercises in realistically hazardous driving conditions. At the same time this course applied objective driver-performance scoring to measure and record the development of safety skills. UDPS applied EVOC-101 to train 355 drivers in 2005 and an additional 598 drivers in 2006. This paper analyzes the data collected by UDPS while using EVOC-101 to train all 953 drivers over this two-year period. 


\section{ISD ANALYSIS}

Instructional Systems Design theory suggests the need to apply several components in effective training processes (Dick, 2004). Four of the essential components include (1) task analysis, (2) incremental skills exercises, (3) performance feedback based on objective measurement, and (4) repetitive practice. The EVOC-101 program incorporated these four components as follows:

\section{Task Analysis}

Trainers at UDPS surveyed hundreds of other EVO trainers, police departments, and state and federal agencies to determine the essential tasks required to negotiate traffic safely in EVO conditions. UDPS used this survey to produce a list of discrete behaviors that officers must do, and not do, when driving in EVO mode. Their task analysis concluded that there were three categories of tasks, and assigned each category a weighted value, as shown in Table 1.

\section{Table 1. Scoring of Tasks by Priorities}

\begin{tabular}{|l|c|l|}
\hline Critical & $5 \mathrm{pts}$ & Tasks affecting vehicle control, safety and risk of collision \\
\hline Priority & $3 \mathrm{pts}$ & Tasks involving uniform traffic code and agency policies and procedures \\
\hline Important & $1 \mathrm{pt}$ & Tasks facilitating arrival at the EVO destination most expeditiously \\
\hline
\end{tabular}

\section{Incremental Skill-Development Exercises}

EVOC-101 lesson plans incorporate sequentially ordered driving scenarios that provide incrementally advancing degrees of complexity. The first EVOC-101 lesson plan that UDPS applied in 2005 is called Thru Intersections. This plan contains 15 scenario exercises, subdivided into three groups of skill levels, including 5 fundamental, 5 intermediate, and 5 advanced scenarios (plus 1 variant). UDPS instructed their trainees in 2005 that after they experienced these 15 exercises they must pass a final exam exercise that included 4 sequential advanced intersections. UDPS told the trainees that passing would require $>80 \%$ score with no more that 2 critical errors. This obligated the trainees to focus on the skills. In 2006 UDPS added another lesson plan called Turn Intersections that added 15 new training exercises that were much like the first lesson plan. However, the new scenarios focused on turning while in the intersections.

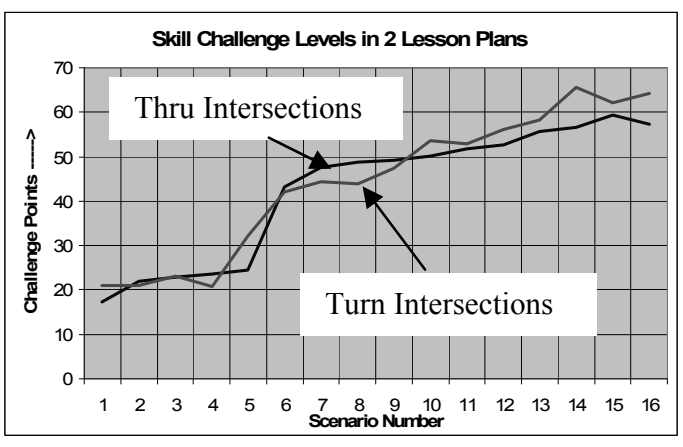

Figure 1. Scenario Challenge Level
Figure 1 illustrates the relative complexity in the sequence of scenarios in these two lesson plans. It indicates that the last scenarios demand 3.5 times more skill than the first. Ultimately, UDPS derived a variety of lesson plans that applied this progression of difficulty. Each variation included 15 exercises using 5 exercises in each of 3 levels of difficulty in the original lesson plans. This variety reduced the number of times that a trainee witnessed any given scenario. 
Equation (1) below characterizes the skill "challenge points" in each scenario, as plotted in the graph shown in Figure 1. There are five elements of complexity used in equation (1) as follows:

- Number of points possible from the tasks in the scenario

- Number of fixed objects along the course

- Number of moving objects presenting potential conflicts

- Number of real conflicts the trainee must recognize and negotiate

- Points assigned to the degree of precision required in vehicle maneuvering

Skill demand level $=$ Totalpts $/ 3+$ NoFO $/ 2+$ NoVehicles + NoConflicts + Precision

\section{Performance Feedback Based on Objective Measurement}

The EVOC-101 program scores each driver in each exercise according to the completion of each required task. Each task comprises a Boolean event. The driver either does or does not complete a task within the boundaries defined for that task. The driver's score at the end of the exercise is simply the sum of points earned for completed tasks divided by the total points possible. Most importantly, the driver receives immediate feedback from the simulator. Most often that comes through the explanation of the trainer/operator. It always includes a summary score at the end of each exercise that (1) lists the performance scored in every task, (2) identifies each specific error, and (3) explains the specific action required to eliminate each error in each exercise.

\section{Repetitive Practice}

The training process typically assigns 4 trainees to each simulator at one time. Trainees take turns driving the simulator for a set of 3 exercises while the other 3 trainees observe. This process gives each trainee 5 different sets of 3 exercises, while they also witness 3 times as many exercises as they drive. As the trainees rotate turns in driving they receive repetitive feedback from 3 sources: (1) objective scoring of the required skills by the system, (2) instructions for improvement by the trainer, and (3) encouragement from peers as they observe the process. The data suggests that the number of cycles in this process predicts the degree of skills achieved.

\section{DATA ANALYSIS}

This analysis focuses primarily on the training benefits achieved through a sequence of 15 training exercises. The core of the training occurs during this sequence. The final test exercises at the end validate the training but they are administered separately and are not part of this analysis.

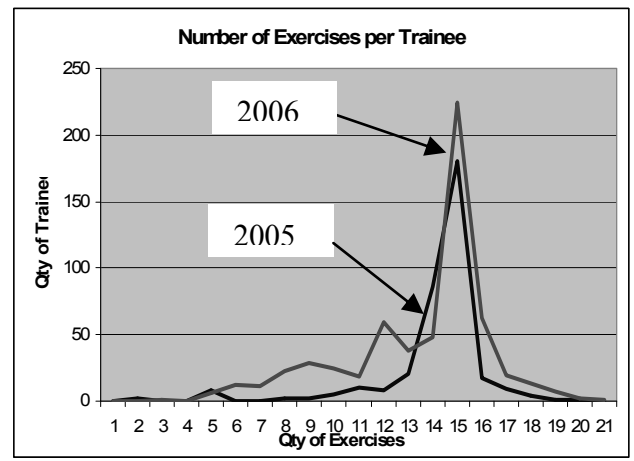

Figure 2. Trainees / Exercise Qty
The data clearly indicates that training in both years was highly biased toward 15 exercises per trainee, but there are some anomalies, particularly in 2006. Detailed review found that a few trainees in both years were credited for driving scenarios that were actually driven by another trainee. This increased one driver's count and reduced another's count in the data. However, that occurs in less than $2 \%$ of the trainees' data sets. Within the 2-year total of $\mathbf{1 2 , 9 8 2}$ trainee exercises, less then $2 \%$ were excluded from this analysis as "invalid attempts." 
Figure 2 shows two secondary "humps" in the distribution for 2006, where a number of trainees experienced 9 and 12 exercises, respectively. Each hump represents a group of trainees who received less than the nominal 15 exercises. These reduced courses occurred when time constraints limited the length of the training sessions.

In 2005 UDPS trained only $\mathbf{2 3}$ in-service (IS) personnel using EVOC-101. In 2006 they trained 150, but they had to abbreviate the IS course because the time previously allotted for IS training did not accommodate the full EVOC-101 program. The number of cadet trainees also increased. That required UDPS to use two additional trainers who had not taught the course in 2005 . The data collected while (1) using new trainers and (2) while varying the course length, enables analyses of the effects that these two issues had on the trainees' skill development.

\section{Cadet Training Analysis}

There were 295 cadets trained in 2005 and 448 trained 2006, which represents an increase of over 50\%. Figure 3 illustrates the average scores for both groups over the course of their training. This shows that the average improvement in scores for all cadets is nearly identical from start to finish for both years. The trainees consistently demonstrate a $25 \%$ increase in score from $65 \%$ to $90 \%$, while the difficulty of the scenarios increases by a multiple of 3.5 times. There is a drop in average scores in exercises 12 and 13 for 2006 because the new lesson plan scenarios presented new conflicts in turns which were not included in the course in 2005. Trainees who made errors while turning encountered more collisions. Since each collision reduces that exercise score to zero, those exercises significantly reduced those trainees' average scores, as well as the average scores for those scenarios.

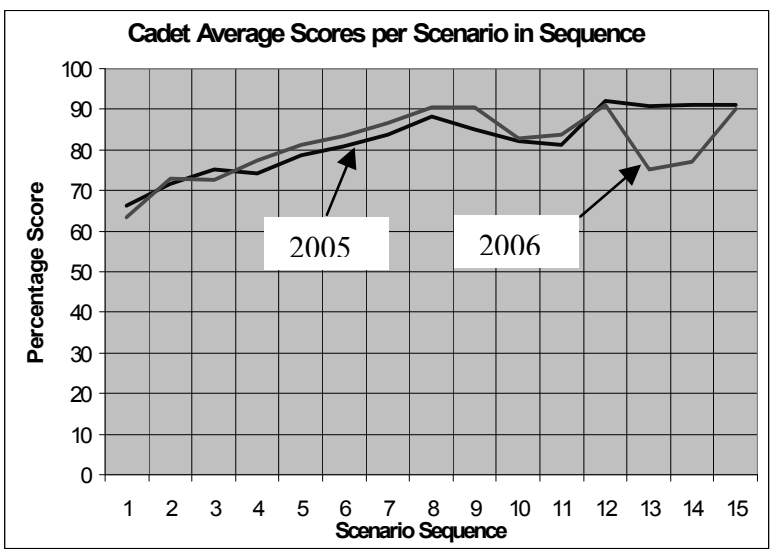

Figure 3. Average Cadet Scores

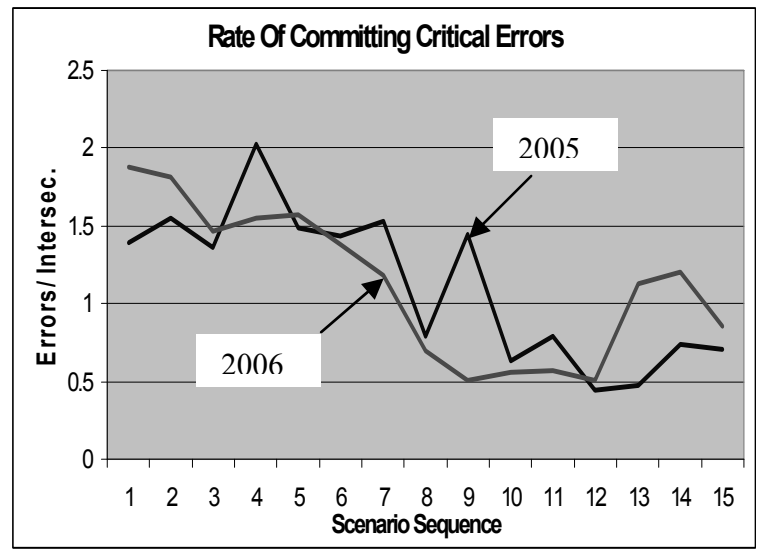

Figure 4. Critical Errors/Intersection

Figure 4 above illustrates the rate of committing critical errors per intersection (CEPI) during the course of the training. Tracking the rate of CEPI appears to be very important because this rate is proportional to the risk for accidents, since by definition every accident is caused by one or more critical errors. The data demonstrates a dramatic net reduction in CEPI during the training in both years. The average rate drops from 1.7 CEPI in easy conditions at the beginning of the course to $0.8 \mathrm{CEPI}$ in conditions that are 3.5 times more challenging at the end of the course. This measure of reduced CEPI clearly reveals an objectively quantified value in this training. 
Further, each trainee's average score over the full course of their training provides a useful measure in determining each trainee's relative position within the overall population of trainees. Figure 5 below illustrates the actual distribution of average scores for cadets in both years. These appear as normal statistical distributions. Each trainee's performance can be analyzed with complete objectivity by simply calculating the normal standard deviation - the $\sigma$ value - in each distribution and then using that $\sigma$ value to make a comparison to the individual's score.

Table 2. Comparative Cadet Statistics

\begin{tabular}{|l|r|r|r|r|}
\hline & \multicolumn{1}{|c|}{ Score } & \multicolumn{1}{c|}{ CEPI } & \multicolumn{1}{c|}{ Time } & Collis. \\
\hline 2006 Ave & 82.1 & 1.073 & 50.9 & 0.031 \\
\hline $2006 \sigma$ & 6.8 & 0.441 & 4.8 & 0.045 \\
\hline & & & & \\
\hline 2005 Ave & 82.1 & 1.142 & 50.3 & 0.020 \\
\hline $2005 \sigma$ & 6.6 & 0.486 & 4.8 & 0.039 \\
\hline
\end{tabular}

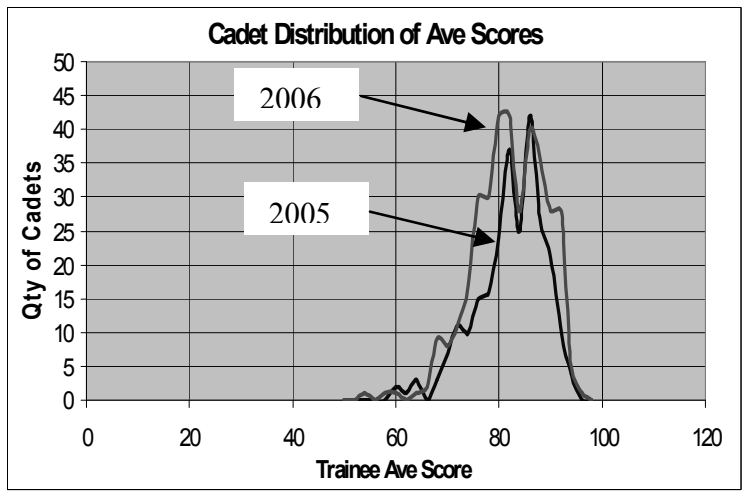

Figure 5. Cadet Scoring Distributions

Table 2 shows that the average score for all of the cadets who experienced 13 to 17 exercises in each year is exactly the same for both years, even though many of the cadets in 2006 experienced some scenarios that were more challenging. The $\sigma$ values for these distributions center about the same average, but there was a wider spread of variance in 2006. There were more collisions. There were 3 per 100 exercises in 2006 compared to 2 per 100 exercises in 2005, but there was a net reduction in the average CEPI in 2006. Apparently, the same number of exercises produced nearly identical average scores. Yet, the more challenging scenarios in 2006 widened the range of average scores between those who caught on quickly and those who needed more practice.

\section{Trainer Influence Analysis}

Three trainers used EVOC-101 to train UDPS cadets in 2006. Figure 6 shows their participation.

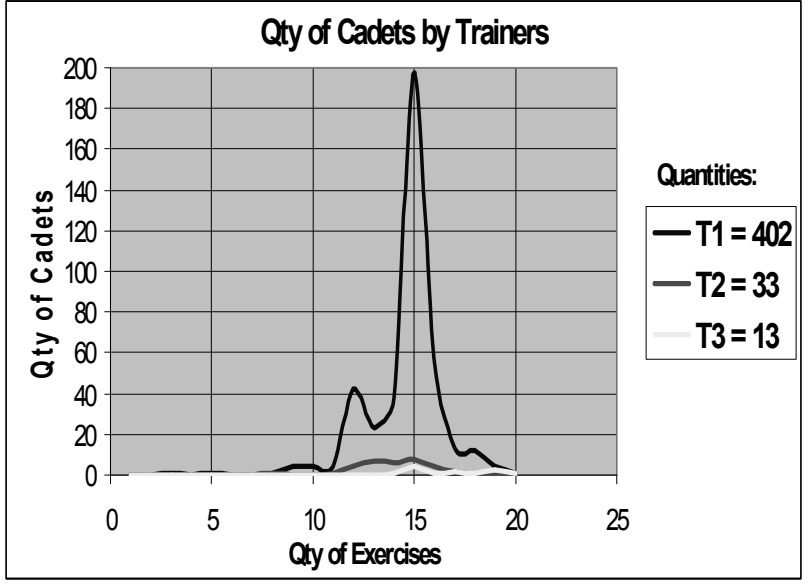

Figure 6. Cadet Performance by Trainer

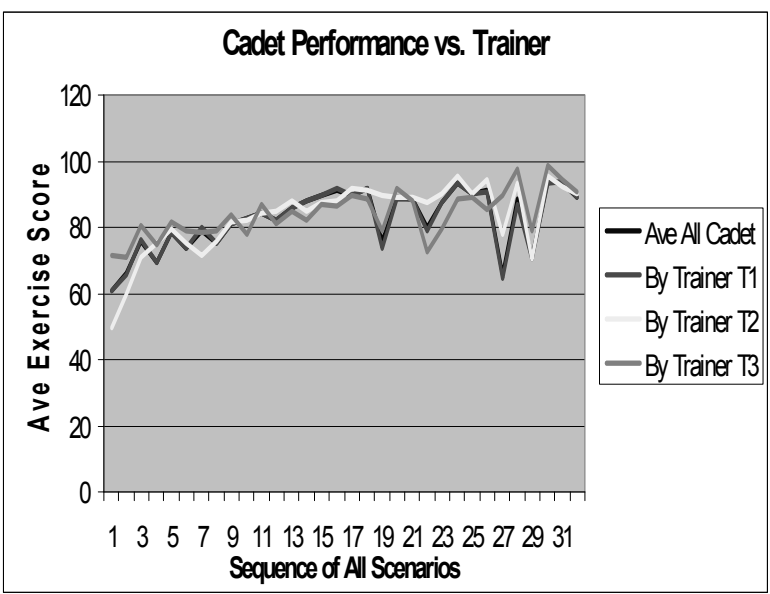

Figure 7. Cadet Distribution by Trainer 
Figure 7 lists each of the 32 scenarios used in the different EVOC-101 lesson plans according to their challenge level. Typically, each cadet drove $1 / 2$ of these scenarios. On average, the trainees experienced 15 exercises, including 5 scenarios in each of the 3 levels of difficulty.

Figure 6 shows that trainer T1 trained nearly 10 times as many cadets as the other two trainers combined, which means that trainers $\mathrm{T} 2$ and $\mathrm{T} 3$ must have acquired considerably less experience using the EVOC-101 program. Nevertheless, Figure 7 shows that all the cadets' performance throughout the course of training followed the same pattern of improvement, independent of the trainer! Apparently, the program provides the training value. While the trainers are required to implement the logistics of the course and provide feedback to motivate the trainees, the trainees' development of skills appears quite independent of differences in these trainers' experience.

\section{Course Length Analysis}

Table 3 itemizes trainee performance according to variations in the number of exercises experienced by different trainees. It uses the three principle groups of trainees identified in Figure 2 whose experience centers about 9, 12, and 15 exercises per trainee, respectively. The variation is primarily due to different allocations of time given for different training sessions.

Table 3. Comparative Training Effect vs. Exercises per Trainee

\begin{tabular}{|l|c|c|c|c|c|}
\hline & Ave Score & CEPI & Time (sec/exrc) & Collisions/exrc & Sample Qty \\
\hline Std. Norm. Ave. & 83.0 & 1.033 & 51.0 & 0.025 & 190 \\
\hline 14-16 Ex/ Trainee & 82.2 & 1.084 & 50.8 & 0.029 & 334 \\
\hline 11-13 Ex/ Trainee & 81.8 & 1.083 & 49.8 & 0.028 & 115 \\
\hline 8-10 Ex/ Trainee & 79.1 & 1.273 & 46.0 & 0.016 & 77 \\
\hline & & & & & \\
\hline Std. Sigma & 6.4 & 0.414 & 4.6 & 0.040 & 190 \\
\hline$\sigma$ for 14-16 Ex/T & 6.8 & 0.445 & 4.7 & 0.044 & 334 \\
\hline$\sigma$ for 11-13 Ex/T & 7.7 & 0.483 & 5.8 & 0.048 & 115 \\
\hline$\sigma$ for 8-10 Ex/T & 8.8 & 0.650 & 6.6 & 0.043 & 77 \\
\hline & & & & & 190 \\
\hline Std. \%-ile & 50.0 & 50.00 & 50.0 & 50.00 & 334 \\
\hline 14-16 Ex/T \%-ile & 48.6 & 48.70 & 50.4 & 48.98 & 115 \\
\hline 11-13 Ex/T \%-ile & 48.0 & 48.72 & 53.6 & 49.17 & 77 \\
\hline 8-10 Ex/T \%-ile & 33.6 & 34.86 & 85.7 & 52.27 & \\
\hline
\end{tabular}

Table 3 also presents a Standard Normal Average performance for reference, using data from a core segment of the population. In this case the standard reference was arbitrarily assigned to be cadets who experienced exactly 15 training exercises. Table 3 itemizes the three other subsets of the population as being those trainees who experienced 8-10,11-13, and 14-16 exercises, respectively. The center band of Table 3 shows that the degree of deviation in the scoring between these four groups increases as the number of exercises decreases. This clearly indicates wider variation in skill levels between the trainees in the groups with fewer exercises. 
Note that the bottom line in Table 3 indicates that those trainees who experienced only 8-10 exercises placed in the lower $35^{\text {th }}$ percentile in this population. In other words, $65 \%$ of the population achieved a higher level of performance than the average of those trainees who received 10 exercises or less. Apparently, the average trainee needs 12 or more practice exercises to attain the majority of the measured value in this training.

\section{SUMMARY}

The second year of driver performance measurements collected by the EVOC-101 training course at UDPS re-affirms all of the findings of the first year. The EVOC-101 tools provide objective measurement of the improvement of driver performance during the training, and they demonstrate tangible value in the training course. While this report does not re-examine all the issues relating to simulator adaptation syndromes (SAS - often misnamed "simulator sickness"), it does report that the occurrence of SAS during the EVOC-101 training in 2006 was still less than the occurrence of drop-out due to discomfort reported by trainees driving real vehicles on the testtrack at UDPS. Most notably, the average rate of critical errors per intersection (CEPI) decreased in 2006, even though the 2006 lesson plans added new exercises with greater complexity.

Further, the data collected by EVOC-101 in 2006 indicates that driver performance improvements achieved during this course of training are essentially independent of the trainer. Apparently, this course incorporates both the tools and the processes required to teach safe EVO operation, provided the trainees practice a sufficient number of exercises to acquire the needed skills.

\section{REFERENCES}

Dick, Walter O., Carey, Lou, \& Carey, James O. (2004). The Systematic Design of Instruction. Allyn \& Bacon.

Hadley, J.A. \& Gibbons, A.S. (2003). Plugging Instruction into Simulation. 2006, Available at http://ntsa.metapress.com/link.asp?id=t4vrm85576181djk. Retrieved Apr 14,

Turpin, D.R. \& Welles, R.T. (2006). Simulator-based Training Effectiveness through Objective Driver Performance Measurement. Proceedings of DSC Asia/Pacific 2006, Tsukuba, Japan.

Turpin, D.R. \& Welles, R.T. (2006). Analysis of Simulator-based Training Effectiveness through Driver Performance Measurement. Proceedings of the Interservice/Industry Training Simulation, and Education Conference (I/ITSEC), No. 2850, Orlando, Florida. 